\title{
Antimicrobial susceptibility of Gram-negative uropathogens isolated from obstetric patients
}

\author{
Whitney E. Jamie, Rodney K. Edwards and Patrick Duff \\ Division of Maternal-Fetal Medicine, Department of Obstetrics and Gynecology, \\ University of Florida College of Medicine, Gainesville, FL
}

\begin{abstract}
Objective: To evaluate the antimicrobial susceptibility of Gram-negative uropathogens isolated from pregnant women.

Methods: We performed a snapshot cohort study of women receiving care in the University of Florida prenatal clinics during March 2000. Subjects with asymptomatic bacteriuria or cystitis were identified and the antimicrobial susceptibility of each pathogen was recorded. Data were analyzed using chi-square, Fisher's exact test and ninety-five percent confidence intervals, as appropriate.
\end{abstract}

Results: Ninety-five positive cultures were identified. Isolates were more often susceptible to trimethoprimsulfamethoxazole (TMP-SMX) $(87 \%)$ and nitrofurantoin (89\%) than to ampicillin $(72 \%)(p<0.03)$. Escherichia coli accounted for $7 \mathrm{I}(75 \%)$ cases and was more often susceptible to nitrofurantoin (I00\%) than to TMP-SMX (87\%) $(p<0.0 \mathrm{I})$. Proteus isolates were all susceptible to TMP-SMX and resistant to nitrofurantoin $(p<0.0 \mathrm{I})$.

Conclusions: Both TMP-SMX and nitrofurantoin are superior to ampicillin for empiric treatment of lower urinary tract infection in pregnant women. Nitrofurantoin is superior to TMP-SMX for treatment of infections caused by E. coli. For suspected or confirmed cases caused by Proteus organisms, TMP-SMX is the preferred agent.

Key words: Pregnancy; Urinary Tract Infection; Antibiotic Resistance; Asymptomatic BACTERIURIA; CYSTITIS

Urinary tract infections (UTI) are the most commonly encountered infections in obstetric patients $^{1}$. These infections can be divided into asymptomatic bacteriuria (ASB), occurring in $5-10 \%$ of patients; acute cystitis, affecting $1-3 \%$ of patients; and pyelonephritis, which complicates $0.5-1.5 \%$ of pregnancies ${ }^{2}$. Most cases of pyelonephritis are the sequelae of untreated, recurrent or inadequately treated lower UTI. Pyelonephritis is the most common non-obstetric cause of hospital admission during pregnancy and can lead to preterm labor, maternal sepsis and acquired respiratory distress syndrome (ARDS) ${ }^{3}$.
Pregnant women diagnosed with ASB or acute cystitis are often treated empirically before the results of culture and sensitivity testing are available ${ }^{4}$. The choice of empiric antibiotic therapy should be based on established patterns of antimicrobial sensitivities in the specific institution. In the past, ampicillin was used extensively as first-line treatment. However, in recent years, the usefulness of ampicillin has been limited because of the growing emergence of bacterial strains resistant to this antibiotic ${ }^{5}$. Currently, the two most commonly chosen therapies are trimethoprimsulfamethoxazole (TMP-SMX) and nitrofurantoin 
monohydrate macrocrystals (hereafter referred to as nitrofurantoin $)^{6}$.

At the University of Florida, TMP-SMX has been the agent of choice due to lower cost and presumed equal efficacy. Unpublished data from our institution showed that as recently as 1996 the resistance of common uropathogens to TMPSMX and nitrofurantoin was equal. However, the cost difference has been narrowing and, more importantly, recent reports suggest there may be increasing resistance to TMP-SMX among common uropathogens ${ }^{7-10}$. As an example, researchers from the University of Washington recently reported that the resistance of Escherichia coli in healthy, nonpregnant women to TMP-SMX increased from $9 \%$ to $18 \%$ from 1992 to $1996^{7}$. The same investigators also reported that resistance of $E$. coli to nitrofurantoin during the same time period was only $0.2 \%$ and did not change over time ${ }^{7}$.

We undertook this study to evaluate which agent should be used for empiric therapy of lower UTI in our obstetric population. The objective of our investigation was to determine the frequency of resistance of common uropathogens to ampicillin, TMP-SMX and nitrofurantoin.

\section{SUBJECTS AND METHODS}

We performed a snapshot cohort study by systematically reviewing all of the prenatal charts in the University of Florida clinic system during March 2000. Therefore, this cohort represents patients at various gestational ages who were receiving care in the system at that time. All cultures from the current pregnancy were included in the review. The investigation was approved by the University of Florida Health Center Institutional Review Board. We removed all patient identifiers from our records once data had been abstracted from subjects' charts.

More than $90 \%$ of our patients are indigent, either uninsured or insured by the Medicaid program and most live in rural areas around Gainesville. Approximately $80 \%$ of patients are Caucasian and most of the remainder are African-American. The vast majority of these patients received prenatal care in the rural county health departments or in the residents' continuity clinics. Approximately 5\% of subjects received care in the faculty private practice.

Patients with a diagnosis of ASB or cystitis were identified. For the purpose of this investigation, asymptomatic bacteriuria was defined as a cleancatch midstream urine culture showing greater than 100000 colonies per milliliter (CPM) of a single Gram-negative uropathogen during routine prenatal screening in an asymptomatic patient. Cystitis was diagnosed in women who had symptoms of frequency, urgency or hesitancy and a urine culture that had greater than 100000 CPM of a single Gram-negative organism. The type of infection (ASB or cystitis), causative organism and antibiotic sensitivity profile were recorded for each positive culture. The antibiotics specifically evaluated were ampicillin, TMP-SMX and nitrofurantoin.

Results were analyzed with descriptive statistics. The uncorrected chi-square test and Fisher's exact test were applied for categorical variables. Ninety-five percent confidence intervals were used for proportions. All statistical tests were twotailed and we considered a $p$-value of $<0.05$ to be statistically significant.

\section{RESULTS}

A total of 95 pregnant women with positive urine cultures meeting the inclusion criteria were identified. Of these women, 72 had ASB and 23 had cystitis. E. coli was isolated from 71 (75\%) subjects. Klebsiella pneumoniae and Proteus mirabilis accounted for eight (8\%) cases each. The remaining cultures were positive for Pseudomonas (two cases), Citrobacter (three cases) and Acinetobacter (three cases).

Table 1 presents the proportions of isolates that were susceptible to ampicillin, TMP-SMX and nitrofurantoin. When considering all Gramnegative organisms combined, susceptibility to TMP-SMX and nitrofurantoin were similar $(p=0.65)$. Bacterial isolates were more likely to be susceptible to either of these agents than to ampicillin $(p<0.03$ for TMP-SMX and $p<0.01$ for nitrofurantoin). When considering only infections caused by E. coli, significantly more of the 
Table I Antibiotic susceptibilities of Gram-negative uropathogens ( $95 \%$ confidence intervals)

\begin{tabular}{lccc}
\hline Organism(n) & Ampicillin & TMP-SMX & Nitrofurantoin \\
\hline Escherichia coli (7I) & $75 \%(64,84)$ & $87 \%(77,93)^{*}$ & $100 \%(95,100)^{*}$ \\
Klebsiella pneumoniae $(8)$ & $25 \%(7,59)$ & $75 \%(41,93)$ & $75 \%(41,93)$ \\
Proteus mirabilis (8) & $100 \%(68,100)$ & $100 \%(68,100)^{*}$ & $0 \%(0,32)^{*}$ \\
Total $(95)$ & $72 \%(62,80)$ & $87 \%(79,92)^{+}$ & $89 \%(81,94)^{+}$ \\
\hline
\end{tabular}

${ }^{*} p<0.0$ I for TMP-SMX versus nitrofurantoin; ${ }^{+} p=0.65$ for TMP-SMX versus nitrofurantoin, but $p<0.03$ for TMP-SMX versus ampicillin and $p<0.01$ for nitrofurantoin versus ampicillin; total includes an additional three cases each caused by Citrobacter and Acinetobacter and two cases caused by Pseudomonas

isolates were susceptible to nitrofurantoin than to TMP-SMX $(p<0.03)$. Klebsiella isolates were equally as likely to be susceptible to TMP-SMX as to nitrofurantoin. All of the Proteus isolates were susceptible to ampicillin and TMP-SMX; none were susceptible to nitrofurantoin $(p<0.01)$. The distribution of bacteria recovered from women with ASB was no different from that recovered from women with acute cystitis.

\section{DISCUSSION}

The results of our investigation confirm earlier studies that demonstrate that the resistance rate of uropathogens to ampicillin is high enough to prevent this drug from being acceptable empiric treatment for lower UTI in pregnancy. We also have shown that, in our population, TMP-SMX and nitrofurantoin are both still effective against almost $90 \%$ of common uropathogens.

For the most common uropathogen, E. coli, nitrofurantoin is superior to TMP-SMX: in our study sample, $100 \%$ of $E$. coli isolates were susceptible to nitrofurantoin. Prior studies have also shown a sensitivity rate of $95-100 \%{ }^{7,10}$. Because 75-90\% of simple UTI in young women are caused by $E$. colit ${ }^{11}$, our clinical guidelines now recommend using nitrofurantoin as empiric therapy for uncomplicated lower UTI in pregnancy.
Nitrofurantoin would be a poor choice if a Proteus infection were suspected. Factors that might raise one's clinical suspicion that a given patient was infected with a strain of Proteus would include a history of oxalate nephrolithiasis, recurrent UTI, chronic instrumentation or indwelling catheters, or immunodeficiency. In addition, Proteus species contain urease, an enzyme that releases ammonia and causes the urinary $\mathrm{pH}$ to be alkaline. The ability of urinary $\mathrm{pH}$ to predict the causal pathogen in cases of lower UTI has not been evaluated. However, nitrofurantoin might not be the best choice for empiric therapy if the urinary $\mathrm{pH}$ is high.

In this investigation, we used in vitro antibiotic susceptibility of isolated urinary pathogens as the primary outcome. Clearly, the outcome that is more important is clinical response to therapy. However, our study only adds to a growing body of evidence that the proportion of uropathogens resistant to TMP-SMX is increasing ${ }^{7-10}$. In addition, the fact that nitrofurantoin works through multiple mechanisms of action reduces the likelihood that bacteria will develop resistance to this antibiotic $^{12}$. Considered collectively, these facts, along with the fact that the cost of nitrofurantoin and TMP-SMX are currently more comparable than in the past, we think that nitrofurantoin is the preferred agent for empiric therapy of lower UTI in pregnant women.

\section{REFERENCES}

1. Gibbs RS, Sweet RL. Maternal and fetal infectious disorders. In Creasy RK, Resnik R, eds. Maternal-Fetal Medicine, 4th edn. Philadelphia: W.B. Saunders Company, 1999;659-724
2. Duff P. Urinary tract infections. Prim Care Update Ob Gyns 1994;1:12-16

3. Duff P. Pyelonephritis in pregnancy. Clin Obstet Gynecol 1984;27:17-31 
4. Vercaigne LM, Zhanel GG. Recommended treatment for urinary tract infection in pregnancy. Ann Pharmacother 1994;28:248-51

5. Dyer IE, Sarkary TM, Dawson JA. Antibiotic resistance in bacterial urinary tract infections, 1991-1997. West J Med 1998;169:265-8

6. Hutt DM. Management of urinary tract infections. N Engl J Med 1994;330:792

7. Gupta K, Scholes D, Stamm WE. Increasing prevalence of antimicrobial resistance among uropathogens causing acute uncomplicated cystitis in women. J Am Med Assoc 1999;281:736-8

8. Manges AR, Johnson JR, Foxman B, et al. Widespread distribution of urinary tract infections caused by a multidrug-resistant Escherichia coli clonal group. N Engl J Med 2001;4:1055-7
9. Wright SW, Wrenn KD, Haynes ML. Trimethoprim-sulfamethoxazole resistance among urinary coliform isolates. J Gen Intern Med 1999;14: 606-9

10. Gupta K, Hooten TM, Wobbe CL, et al. The prevalence of antimicrobial resistance among uropathogens causing acute uncomplicated cystitis in young women. Int J Antimicrob Agents 1999;11: 305-8

11. Hooton JM, Stamm WE. Diagnosis and treatment of uncomplicated urinary tract infection. Infect Dis Clin North Am 1997;11:551-8

12. Guay DR. An update on the role of nitrofurans in the management of urinary tract infections. Drugs 2001;61:353-64

ReCEIVED 12/03/01; ACCEPTED 03/19/02 


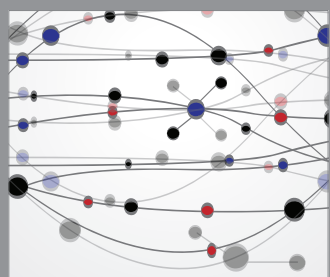

The Scientific World Journal
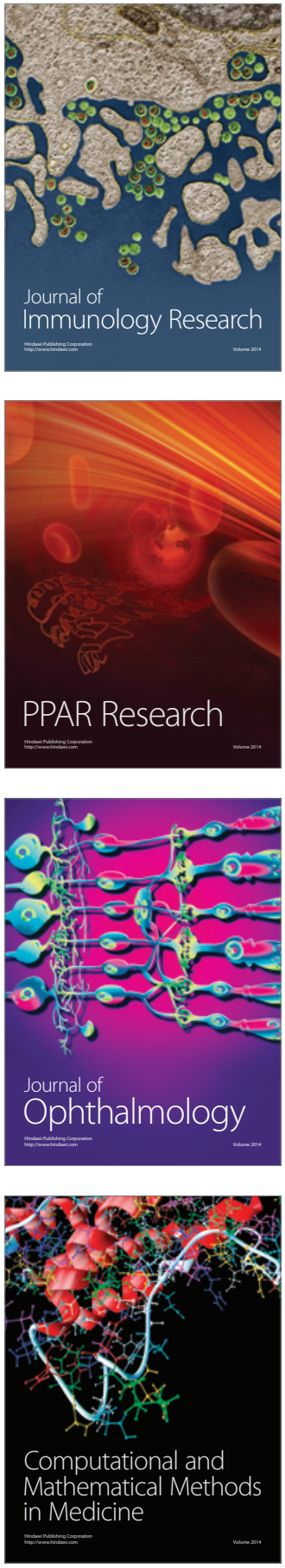

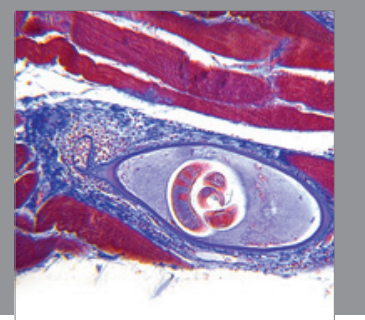

Gastroenterology

Research and Practice
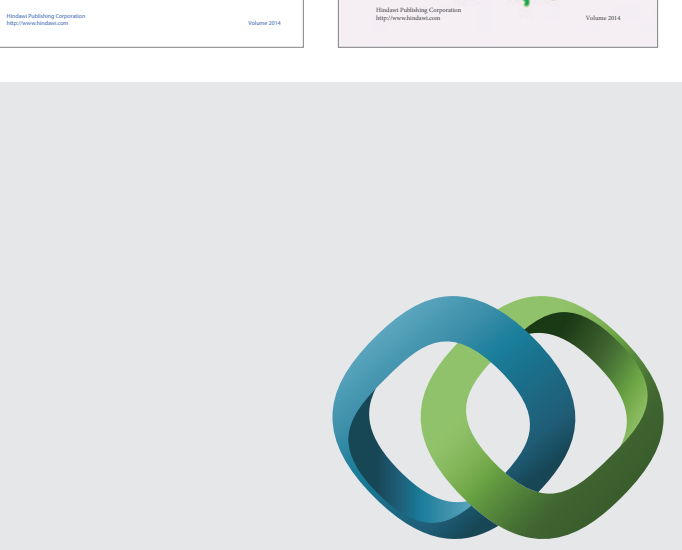

\section{Hindawi}

Submit your manuscripts at

http://www.hindawi.com
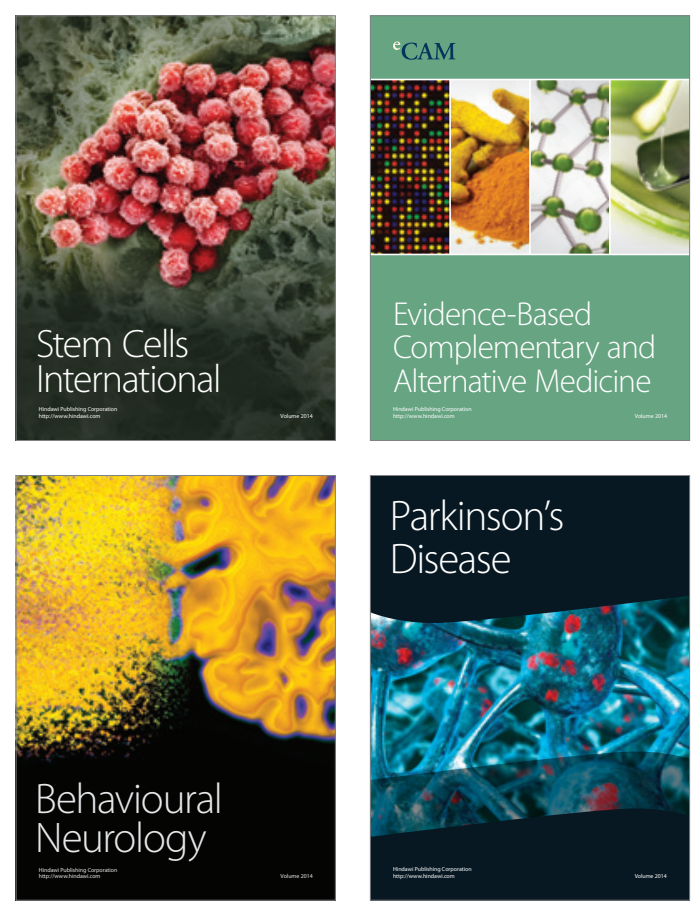

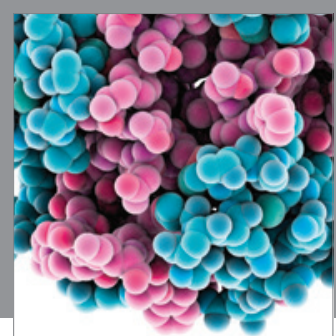

Journal of
Diabetes Research

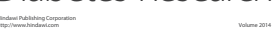

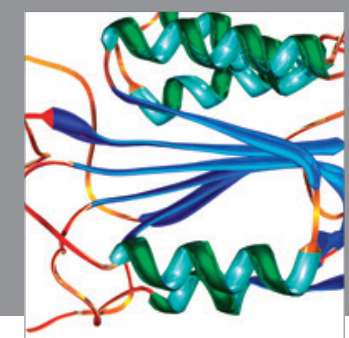

Disease Markers
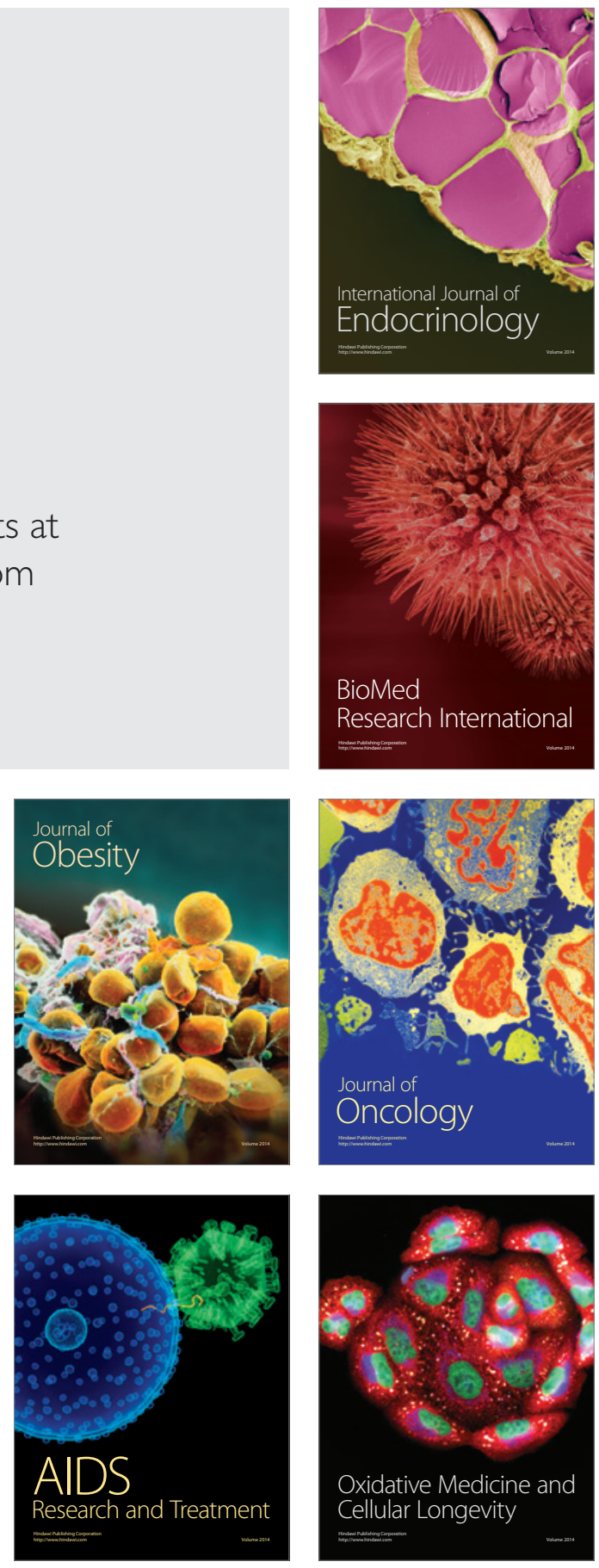